\title{
Expression of HYOU1 via reciprocal crosstalk between NSCLC cells and HUVECs control cancer progression and chemoresistance in multicellular tumor spheroids
}

\author{
Minji Lee \\ Institut Pasteur de Coree \\ Yeonhwa Song \\ Institut Pasteur de Coree \\ Inhee Choi \\ Institut Pasteur de Coree \\ Su-Yeon Lee \\ Institut Pasteur de Coree \\ Sanghwa Kim \\ Institut Pasteur de Coree \\ Se-Hyuk Kim \\ Institut Pasteur de Coree \\ Jiho Kim \\ Institut Pasteur de Coree \\ Haengran Seo ( $\nabla$ shr1261@ip-korea.org ) \\ Institut Pasteur de Coree
}

\section{Research}

Keywords: Hypoxia up-regulated protein1 (HYOU1), Lung cancer, Multicellular Tumor Spheroids (MCTS), Endothelial cells (ECs), chemoresistance

Posted Date: August 20th, 2020

DOI: https://doi.org/10.21203/rs.3.rs-61542/v1

License: (c) (i) This work is licensed under a Creative Commons Attribution 4.0 International License.

Read Full License 


\section{Abstract \\ Background}

Among all cancer types, lung cancer ranks highest worldwide in terms of both incidence and mortality. The crosstalk between lung cancer cells and their tumor microenvironment (TME) has begun to emerge as the "Achilles heel" of the disease and thus constitutes an attractive target for anticancer therapy. We previously revealed that crosstalk between lung cancer cells and endothelial cells (ECs) induces chemoresistance in multicellular tumor spheroids (MCTSs).

\section{Methods}

In this study, we idientified that factors secreted in response to crosstalk between ECs and lung cancer cells play pivotal roles in the development of chemoresistance in lung cancer spheroids.

\section{Results}

We determined that the expression of hypoxia up-regulated protein 1 (HYOU1) in lung cancer spheroids was increased by factors secreted in response to crosstalk between ECs and lung cancer cells. Direct interaction between lung cancer cells and ECs also caused an elevation in the expression of HYOU1 in MCTSs. Inhibition of HYOU1 expression not only suppressed stemness and malignancy, but also facilitated apoptosis and chemosensitivity in lung cancer MCTSs. Inhibition of HYOU1 expression also significantly increased the expression of interferon signaling components in lung cancer cells. Moreover, the activation of the PI3K/AKT/mTOR pathway was involved in the HYOU1-induced aggression of lung cancer cells.

\section{Conclusion}

Taken together, our results identify HYOU1, which is induced in response to crosstalk between ECs and lung cancer cells within the TME, as a potential therapeutic target for combating the aggressive behavior of cancer cells.

\section{Introduction}

Lung cancer is one of most common cancer types and is the leading cause of cancer death worldwide. Because lung cancer cells often exhibit abnormally high expression of epidermal growth factor receptor (EGFR) or mutations in the EGFR gene, EGFR inhibitors are currently used as the first-line treatment for advanced lung cancer [1-3]. However, because treatment with EGFR inhibitors can lead to the development of mutations conferring resistance to such treatments (e.g., T790M), these drugs have limited therapeutic efficacy in lung cancer [4,5]. Anaplastic lymphoma kinase [6, 7], ROS proto-oncogene1 
$[8,9]$, neurotrophic receptor tyrosine kinases [10-12] and the B-Raf proto-oncogene $[13,14]$ have also emerged as possible therapeutic targets for lung cancer, but drugs based on these targets are less common, and further study of their side effects is still required. Hence, the development of targeted drugs has not yet significantly improved the prognoses of patients with lung cancer.

Recent studies have shown that lung tumor heterogeneity is a major cause of treatment failure due to chemoresistance $[15,16]$. In lung cancers, tumors develop concurrently with the tumor microenvironment (TME), which includes the vasculature, infiltrating immune cells, stromal fibroblasts, signaling molecules, and extracellular matrix surrounding the tumor [17], as evidenced by regions of aberrant angiogenesis, desmoplasia, acidosis, and hypoxia [18]. Within the TME, endothelial cells (ECs) have attracted attention as a key player in cancer progression due to their association with tumor cell angiogenesis, proliferation, and invasion, which have all been linked to the EC-mediated expression of various angiogenic factor receptors and remodeling of the extracellular matrix [19]. [19]. In our previous study, we elucidated that the direct interaction between lung cancer cells and ECs induces chemo- and radioresistance by facilitating the endothelial-to-mesenchymal transition (EndMT) in lung cancer cells [20-22].

Utilizing three-dimensional (3D) multicellular culture systems and modeling tumor interactions with stromal components are essential to establishing more clinically relevant tumor models [20, 23, 24]. Therefore, we used multicellular tumor spheroids (MCTSs) to identify factors that induce chemoresistance in response to crosstalk between lung cancer cells and ECs.

Here, we identified hypoxia upregulated protein 1 (HYOU1) as a factor secreted in response to crosstalk between ECs and lung cancer cells, and sought to elucidate its functional role in lung cancer chemoresistance and tumor growth. HYOU1, also known as oxygen-regulated protein 150 and glucoseregulated protein 170 , is a member of the heat shock protein 70 family and play important roles in hypoxia/ischemia. HYOU1 is crucial for the processing and maturation of vascular endothelial growth factor A during angiogenesis. Extracellular HYOU1 from tumor cells acts as an immunomodulator in the TME [25-28], and expression of HYOU1 has been correlated with poor prognoses in patients with certain cancers, including breast cancer [29], nasopharyngeal carcinoma [30], renal cancer and thyroid cancer [31]. Further, HYOU1 upregulation has been linked to chemoresistance in various tumors [31-33]. However, the functions of HYOU1 in lung cancer remain largely unknown.

In this study, we investigated the effects of HYOU1 upregulation on the progression and chemoresistance of MCTSs. Based on our observations, we also discuss future therapeutic opportunities for lung cancer.

\section{Materials And Methods}

\section{Cell line and cell culture}

$\mathrm{NCl}-\mathrm{H} 460$ cells, A549 cells, H1299 cells, and PC9 cells were obtained from the Korean Cell Line Bank. Human umbilical vein endothelial cells (HUVECs) were obtained from PromoCells (Heidelberg, Germany). 
The cells were maintained at $37{ }^{\circ} \mathrm{C}$ in a humidified atmosphere of $5 \% \mathrm{CO}_{2} . \mathrm{H} 460$ cells, and $\mathrm{H} 1299$ cells were cultured in Roswell Park Memorial Institute medium (RPMI 1640; Welgene, Korea) supplemented with 10\% fetal bovine serum (FBS; Gibco, Grand Island, NY, USA), 1\% penicillin-streptomycin (P/S; Gibco, Grand Island, NY, USA) (complete medium). A549 cells were cultured in Dulbecco's Modified Eagle medium (DMEM; Welgene, Korea) supplemented with 10\% FBS and 1\% P/S. HUVECs were cultured in endothelial basal medium (EBM; PromoCells, Heidelberg, Germany) supplemented with $10 \%$ FBS and $0.5 \% \mathrm{P} / \mathrm{S}$.

\section{Generation of lung cancer spheroids with conditioned medium}

HUVECs, lung cancers (NCl-H460 cells or A549 cells), and lung cancers with HUVECs were cultured in 2D conditions using the same number of cells and amount. Conditioned medium (CM) from cultured HUVECs, lung cancers ( $\mathrm{NCl}-\mathrm{H} 460$ cells or A549 cells), and lung cancers with HUVECs were collected when the cells reached $70-90 \%$ confluence, and passed through a $0.45-\mu \mathrm{M}$ pore filter (Millipore, Billerica, MA, USA) to eliminate debris. Lung cancer cells ( $\mathrm{NCl}-\mathrm{H} 460$ cells, A549 cells, or $\mathrm{H} 1299$ cells) were seeded at a density of $6 \times 10^{3}$ cells/well in 96-well round-bottomed ultra-low attachment microplates (Corning, NY, USA) with $80 \mu$ l of filtered 2D-CM for $48 \mathrm{~h}$.

\section{Generation of lung cancer spheroids with HUVECs}

Lung cancer cells (NCl-H460 cells, or A549 cells) with or without HUVECs at a ratio of 5:5, or 7:3 were seeded at a density of $6 \times 10^{3}$ cells/well in 96-well round-bottomed ultra-low attachment microplates (Corning, NY, USA) with $80 \mu$ of complete medium for $72 \mathrm{~h}$.

\section{siHYOU1 transfection in lung cancer cells}

Lung cancer cells ( $\mathrm{NCl}-\mathrm{H} 460$ cells, A549 cells, or H1299 cells) were incubated in complete medium when the cells reached $60-70 \%$ confluence in 100-mm dish (Nunc, Thermo Scientific, Waltham, MA, USA) at $37^{\circ} \mathrm{C}$ incubator for $24 \mathrm{~h}$. After washing for two times, $6 \mu \mathrm{M}$ siRNA targeting HYOU1 (Dharmacon, Lafayette, CO, USA) and 40 pM lipofectamine RNAiMAX reagent (Invitrogen, Eugene, OR, USA) diluted in Opti-MEM (Gibco, Grand Island, NY, USA) medium were treated in fresh medium without supplements at $37^{\circ} \mathrm{C}$ incubator for $24 \mathrm{~h}$. After washing for two times, $0.05 \%$ trypsin-ethylenediaminetetraacetic acid (EDTA) (Gibco, Grand Island, NY, USA) was treated for 3 min at $37^{\circ} \mathrm{C}$ incubator, and then added equal amounts of complete medium. The cell suspensions were centrifuged at 1,300 rpm for $3 \mathrm{~min}$, and then the cells were counted.

\section{Cell death detection in spheroid}

Non-specific SiRNA (SiCont) or HYOU1 SiRNA (SiHYOU1) transfected for $24 \mathrm{~h}$ or 5, 10, 20, or $40 \mu \mathrm{M}$ of gefitinib and cisplatin (all from Sigma-Aldrich, St. Louis, MO, USA) treated for $48 \mathrm{~h}$, lung cancer cells ( $\mathrm{NCl}-$ H460 cells, A549 cells, or H1299 cells) with or without HUVECs were seeded at a density of $6 \times 10^{3}$ cells/well in 96-well round-bottomed ultra-low attachment microplates (Corning, NY, USA). After 2 or 3 
days, the spheroids cell death was detected using the cell-impermeant viability indicator ethidium homodimer-1 (EthD-1; Invitrogen, Eugene, OR, USA). EthD-1 is a high-affinity nucleic acid stain that fluoresces weakly until bound to DNA, whereupon it emits red fluorescence (excitation/ emission maxima $\sim 528 / 617 \mathrm{~nm}$ ). Spheroids were incubated in $4 \mu \mathrm{M}$ EthD-1 in complete medium for $30 \mathrm{~min}$ in a $37^{\circ} \mathrm{C}$ incubator, and images were obtained and the intensity of EthD-1 fluorescence measured using the Operetta ${ }^{\circledR}$ High Content Screening System (Perkin Elmer, Waltham, MA, USA). Fluorescent intensity analysis was performed using the Harmony software (Perkin Elmer, Waltham, MA, USA).

\section{Microarray analysis}

Global gene expression analysis was performed using Affymetrix GeneChip ${ }^{\circledR}$ Human Gene 2.0 ST Arrays. Total RNA from non-specific SiRNA (SiCont) or HYOU1 SiRNA (SiHYOU1) transfected NCl-H460 cells was isolated using the RNeasy Mini kit (Qiagen, Hilden, Germany). RNA quality was assessed using an Agilent 2100 Bioanalyser using the RNA 6000 Nano Chip (Agilent Technologies), and the quantity was determined using a Nanodrop-1000 Spectrophotometer Thermo Scientific). We used $300 \mu \mathrm{g}$ of each RNA sample as input for the Affymetrix procedure, as recommended in the manufacturer's protocol (http:// www.affymetrix.com). Briefly, $300 \mathrm{ng}$ of total RNA from each sample was converted to double-stranded cDNA using a random hexamer incorporating a T7 promoter, and amplified RNA (cRNA) was generated from the double-stranded cDNA template though an in vitro transcription (IVT) reaction and purified using the Affymetrix sample cleanup module. cDNA was regenerated through randomly primed reverse transcription using a dNTP mix containing dUTP. The cDNA was then fragmented by uracil-DNA glycosylase (UDG) and apurinic/apyrimidinic endonuclease (APE 1) restriction enzymes, and end-labeled via a terminal transferase reaction incorporating a biotinylated dideoxynucleotide. Fragmented endlabeled cDNA was hybridized to the GeneChip ${ }^{\circledR}$ Human Gene $2.0 \mathrm{ST}$ array for $17 \mathrm{~h}$ at $45^{\circ} \mathrm{C}$ and $60 \mathrm{rpm}$, as described in the Gene Chip Whole Transcript (WT) Sense Target Labeling Assay Manual (Affymetrix). After hybridization, the chips were stained and washed in a Genechip Fluidics Station 450 (Affymetrix) and scanned using a Genechip Array scanner 3000 7G (Affymetrix). The expression intensity data were extracted from the scanned images using Affymetrix Command Console software, version 1.1, and stored as CEL files.

\section{Immunocytochemistry in lung cancer cells co-cultured with HUVECs spheroid}

HUVECs were stained cell-labeling solution DiD (Molecular Probes, Eugene, Oregon, USA). DiD allows cell populations to be marked in distinctive fluorescent colors for identification, whereupon it emits red fluorescence (absorption/ emission maxima 644/665 nm). HUVECs were incubated at a density of 1.5 $\times 10^{5}$ cells in $1 \%$ DiD in complete medium for $20 \mathrm{~min}$ in a $37^{\circ} \mathrm{C}$ incubator. To generate spheroids, lung cancer cells (NCl-H460 cells and A549 cells) cultured with HUVECs at a ratio of 7:3 were seeded at a density of $6 \times 10^{3}$ cells/well in 96-well round-bottomed ultra-low attachment microplates (Corning, NY, USA) for 3 days at $37{ }^{\circ} \mathrm{C}$ in a humidified atmosphere of $5 \% \mathrm{CO}_{2}$. After 3 days, spheroids were fixed in $4 \%$ paraformaldehyde (PFA) (Biosesang, Gyeonggi-do, Korea) for $24 \mathrm{~h}$ and washed three times with 
Dulbecco's Phosphate-Buffered Saline (DPBS; Welgene, Korea), and then 0.1\% Triton X-100 (SigmaAldrich, St. Louis, MO, USA) for $30 \mathrm{~min}$ at room temperature. After washing with DPBS three times, the spheroids were incubated with rabbit polyclonal anti-HYOU1 (1:100, Cell Signaling Technology, Danvers, MA, USA) in DPBS with 10\% normal goat serum (Vector Laboratories, Burlingame, CA, USA) for $16 \mathrm{~h}$ at $4{ }^{\circ} \mathrm{C}$, and then washed three times for $10 \mathrm{~min}$ with DPBS. The secondary antibodies used for staining were: goat anti-mouse Alexa ${ }^{\circledR}$ Fluor 488 and goat anti-rabbit Alexa ${ }^{\circledR}$ Fluor 546 (1:200, Invitrogen, Eugene, OR, USA). Secondary antibodies were incubated in $1 \%$ BSA for $1 \mathrm{~h}$ at room temperature in the dark. After washing with DPBS three times in 5 min, the nuclei were stained with Hoechst 33342 (Invitrogen, Eugene, OR, USA) for $10 \mathrm{~min}$ and then washed three times. Fluorescent images were obtained using an Operetta ${ }^{\circledR}$ High Content Screening System (Perkin Elmer, Waltham, MA, USA) with a $10 \times$ objective and the merge in $3 \mathrm{D}$ images were combined 40 images taken at each $5 \mu \mathrm{m}$ from - $50 \mu \mathrm{m}$ until $145 \mu \mathrm{m}$ to get Z-stack images.

\section{Western blot}

2D or 3D cells were lysed using radioimmunoprecipitation assay (RIPA) buffer (3 M, Seoul, Korea) and boiled with $5 \times$ sample buffer (Biosesang, Gyeonggi-do, Korea) for $10 \mathrm{~min}$. Cell lysates were separated by 8-15\% sodium dodecyl sulfate-polyacrylamide gel electrophoresis (SDS-PAGE) and transferred to a nitrocellulose (NC) membrane (Pall Corporation, Port Washington, NY, USA). A blocking step was performed for 30 min at room temperature with $5 \%$ skim milk in Tris-buffered saline/Tween 20 (TBST) buffer. After washing for three times in 10 min with TBST buffer, the NC membranes were incubated with mouse monoclonal anti-CD133 (W6B3C1; Miltenyi Biotec, Bergisch Gladbach, Germany), rabbit polyclonal anti-Collagen I (Novus Biologicals, Centennial, Colorado, USA), mouse monoclonal anti-p53 (DO-2; Santa Cruz, California, USA), mouse monoclonal anti-MetRS/MARS (MARSD10B4), rabbit monoclonal antiCARS (EPR7121), mouse monoclonal anti-HIF-1 alpha (mgc3), rabbit polyclonal anti-N Cadherin, mouse monoclonal anti-Vimentin (RV202), rabbit monoclonal anti-alpha smooth muscle Actin (E184) (all from Abcam, Cambridge, UK), rabbit polyclonal anti-HYOU1, rabbit monoclonal anti-phospho-Akt (Ser473) (D9E), rabbit monoclonal anti-PERK (D11A8), rabbit monoclonal anti-ATF-6 (D4Z8V), rabbit monoclonal anti-IRE1a (14C10), rabbit monoclonal anti-cleaved Caspase-3 (Asp175) (5A1E), rabbit monoclonal anticleaved PARP (Asp214) (D64E10), rabbit polyclonal anti-phospho-p44/42 MAPK (Erk1/2) (Thr202/Tyr204) (all from Cell Signaling Technology, Danvers, MA, USA), mouse monoclonal antiinterferon-alpha, goat polyclonal anti-interferon-beta, goat polyclonal anti-interferon-gamma (all from R\&D systems, northeast Minneapolis, USA), and mouse monoclonal anti- $\beta$-actin (Sigma-Aldrich, St. Louis, MO, USA) for $16 \mathrm{~h}$ at $4{ }^{\circ} \mathrm{C}$. After washing for three times in $10 \mathrm{~min}$ with TBST buffer, the membranes were incubated with horseradish peroxidase (HRP)-conjugated secondary antibody (Cell Signaling Technology, Danvers, MA, USA), and the specific bands were visualized by enhanced chemiluminescence (ECL; Thermo Scientific, Waltham, MA, USA).

\section{Stem Cell Spheroid formation in HYOU1 SiRNA transfected lung cancer cells}


Non-specific SiRNA (SiCont) or HYOU1 SiRNA (SiHYOU1) transfected lung cancer cells (NCl-H460 cells, A549 cells, or $\mathrm{H} 1299$ cells) were seeded in low-attachment 6-well plates (Corning, NY, USA) at a density of $3 \times 10^{3}$ cells/well for 7 days. The stem cell permission media was composed of DMEM/F12 (Gibco, Grand Island, NY, USA) supplemented with $1 \times$ B27 (Invitrogen, Eugene, OR, USA), $20 \mathrm{ng} / \mathrm{mL}$ basic fibroblast growth factor (bFGF; Invitrogen, Eugene, OR, USA), $20 \mathrm{ng} / \mathrm{mL}$ epidermal growth factor (EGF; Invitrogen, Eugene, OR, USA), and $25 \mu \mathrm{g} / \mathrm{mL}$ insulin (Sigma-Aldrich, St. Louis, MO, USA). After incubation, the spheroids were observed using Operetta HCS system (Perkin Elmer, Waltham, MA, USA).

\section{Colony formation assay}

For colony formation assays, the lung cancer cells were transfected with SiCont or SiHYOU1. Then, the transfected cells were harvested and replaced at a density of $5 \times 10^{2}$ or $1 \times 10^{3}$ cells/6-well plate (Corning, NY, USA). The medium were changed every three days. Two weeks later, the colonies were washed with DPBS for two times and fixed in $4 \%$ paraformaldehyde (Biosesang, Gyeonggi-do, Korea) for $24 \mathrm{~h}$ at $4{ }^{\circ} \mathrm{C}$. After washing for two times, the colonies were stained with $0.5 \%$ crystal violet (Sigma-Aldrich, St. Louis, MO, USA) in $20 \%$ methanol and washed until background appears light. Subsequently the colonies were photographed by an Eclipse TS100 microscope (Nikon, Tokyo, Japan) and counted.

\section{Cell migration assay}

For cell migration assays, the lung cancer cells ( $\mathrm{NCl}-\mathrm{H} 460$ cells and A549 cells) were transfected with non-specific SiRNA (SiCont) or HYOU1 SiRNA (SiHYOU1). Then, the treated cells were harvested and replaced at a density of $7 \times 10^{5}$ cells/6-well plate (Corning, NY, USA). After 1 day, the cells in the monolayer were vertically scratched using a $200 \mu$ lip (Axygen, Corning, NY, USA) and incubated at $37^{\circ} \mathrm{C}$ in a humidified atmosphere of $5 \% \mathrm{CO}_{2}$. After incubation for 24 or $93 \mathrm{~h}$, images of cell migration were obtained by an Eclipse TS100 microscope (Nikon, Tokyo, Japan).

\section{Drug treatment in lung cancer cells}

Lung cancer cells ( $\mathrm{NCl}-\mathrm{H} 460$ cells and $\mathrm{H} 1299$ cells) were seeded in complete medium when the cells reached $60-70 \%$ confluence in 6-well plate (Corning, NY, USA) at $37^{\circ} \mathrm{C}$ in a humidified atmosphere of $5 \%$ $\mathrm{CO}_{2}$ for. The cells were treated with 0.1 or $1 \mu \mathrm{M}$ mTOR inhibitors, Torin2, WYE-125132, and PI3K inhibitors, GDC0032, PKI-402 (all from Selleckchem, Houston, TX, USA). After 1 day, cells were harvested.

\section{Statistical analysis}

All experiments were performed at least three times. The data are shown as the mean \pm standard deviation (SD). A Student's t-test was used to assess statistically significant differences in excel. The significances were considered respectively with $P$ values of $P<0.05, P<0.01$, and $P<0.001$.

\section{Results}




\section{Crosstalk between ECs and lung cancer cells causes chemoresistance in lung cancer spheroids}

We began by investigating whether interactions between ECs and lung cancer cells cause chemoresistance in lung cancer spheroids. First, we collected conditioned media (CM) from three different cell cultures: human umbilical vein endothelial cells (HUVECs/ECs), non-small cell lung cancer (NSCLC) cells (NCl-H460 or A549), and lung cancer cells co-cultured with HUVECs. We then added the three different $\mathrm{CM}$ to lung cancer spheroids in $3 \mathrm{D}$ cell culture conditions. We next compared the sensitivities of spheroids cultured in each medium to two anticancer drugs (gefitinib and cisplatin) [Figure $1-\mathrm{A}]$.

To detect cell death in the spheroids, we used the nucleic acid stain EthD-1, which emits fluorescence upon binding the DNA of dead cells. Treatment with the EGFR inhibitor gefitinib significantly reduced cell survival in lung cancer spheroids grown in CM from cultured HUVECs or lung cancer cells, whereas spheroids grown in CM from lung cancer cells co-cultured with HUVECs showed strong resistance to gefitinib [Figure 1-B].

We also evaluated the efficacy of cisplatin, a member of the platinum-based antineoplastic family of drugs, under the same conditions. Cisplatin also sufficiently increased the intensity of EthD-1 staining in lung cancer spheroids grown in CM from cultured HUVECs or lung cancer cells. However, cisplatin did not alter cancer cell viability in spheroids grown in CM from lung cancer cells co-cultured with HUVECs [Figure $1-\mathrm{C}]$.

Taken together, these results suggest that factors secreted in response to crosstalk between ECs and lung cancer cells may play pivotal roles in chemoresistance in lung cancer spheroids.

HYOU1 expression is increased by factors secreted in response to crosstalk between ECs and lung cancer cells

We next sought to identify the factors inducing chemoresistance in lung cancer spheroids grown in $\mathrm{CM}$ from lung cancer cells co-cultured with HUVECs. To accomplish this, we subjected spheroids generated via the procedure shown in Fig. 1A to microarray analysis.

Analyses of the functional enrichment of genes with an absolute change of greater than 2-fold were performed using FunRich software. The microarray data revealed that 42 genes were significantly enriched and 103 genes were depleted in lung cancer spheroids grown in CM from lung cancer cells cocultured with HUVECs compared to spheroids grown in CM from lung cancer cells or HUVECs alone [Figure 2-A, Supplementary Table I].

Further, spheroids grown in CM from lung cancer cells co-cultured with HUVECs exhibited a greater than 1.5-fold increase in the expression of genes involved in gene expression, tRNA aminoacylation, and the unfolded protein response (UPR), whereas the expression of genes involved in the cell cycle and DNA 
replication were decreased [Figure 2-B]. In terms of biological processes, we observed the enriched expression of genes involved in the protein metabolism and energy pathways, whereas we observed decreased expression of genes involved in cell communication and nucleic acid metabolism [Figure 2-C].

Thirteen genes (AARS, CARS, CASC5, CENPE, CENPQ, CENPU, GFPT1, HYOU1, IARS, MARS, MCM10, SARS, SGOL2) were detected in all three analyses performed (DAVID, FUNRICH, and GSEA). Because gene expression related to tRNA aminoacylation and the UPR was significantly enriched in spheroids grown in CM from lung cancer cells co-cultured with HUVECs, we focused our efforts on aminoacyl-tRNA synthetase and HYOU1.

Western blot analysis showed that aminoacyl-tRNA synthetase protein expression in lung cancer spheroids was not changed by factors secreted in response to crosstalk between ECs and lung cancer cells. As the PI3K/AKT signaling pathway plays a major role in cell proliferation, cell survival, and invasion in cancer, we next investigated the effects of HYOU1 on PI3K/AKT pathway components. HYOU1 protein expression and AKT phosphorylation at Ser473 were increased in H460 spheroids grown in CM from lung cancer cells co-cultured with HUVECs relative to H460 spheroids grown under normal conditions [Figure 2-D]. This result prompted us to focus on functional roles of HYOU1 in TME of lung cancer.

\section{Reciprocal crosstalk between NSCLC cells and HUVECs causes increased HYOU1 expression in MCTSs}

We further sought to ascertain whether the direct interaction between ECs and lung cancer cells affects HYOU1 expression in MCTSs. To accomplish this, we co-cultured lung cancer spheroids with ECs and/or lung cancer cells (NCl-H460 or A549) in 3D cell culture. Spheroids co-cultured with both ECs and lung cancer cells showed enhanced compactness compared to spheroids cultured with lung cancer cells alone [Figure 3-A]. Using the technique described in [Figure 2D], we next estimated HYOU1 expression and $\mathrm{PI} 3 \mathrm{~K} / \mathrm{AKT}$ signaling activation in these spheroids. We observed increased HYOU1 expression and AKT phosphorylation (Ser473) in spheroids co-cultured with ECs and lung cancer cells compared to spheroids cultured with lung cancer cells alone.

Because HYOU1 is a well-known component of the endoplasmic reticulum (ER) chaperone network, we also explored whether induction of HYOU1 is dependent on the canonical UPR pathway by inducing ER stress in spheroids co-cultured with ECs and lung cancer cells. Unexpectedly, we observed decreased expression of the three major ER stress sensor proteins [inositol-requiring enzyme-1 (IRE1), PKR-like ER kinase, and activating transcription factor-6 (ATF6)] in spheroids co-cultured with ECs and lung cancer cells [Figure 3-B]. These results indicate that the increased HYOU1 expression observed in spheroids cocultured with ECs and lung cancer cells is unrelated to the ER stress response.

Next, we investigated which cells express more HYOU1 upon the co-culture of spheroids with ECs and lung cancer cells. To this end, we detected HYOU1 expression and localization via multilayer image 
acquisition using fluorescence microscopy in lung cancer spheroids ( $\mathrm{NCl}-\mathrm{H} 460$ or A549) grown with HUVECs. Interestingly, HYOU1 expression was only observed in lung cancer spheroids and absent in HUVECs [Figure 3-C].

Our previous study revealed that crosstalk between NSCLC cells and HUVECs induced strong chemoresistance in MCTSs [20]. In the present study, we investigated the effects of HYOU1 on the chemoresistance induced by the interaction between lung cancer cells and HUVECs in MCTSs. We generated tumor spheroids with suppressed HYOU1 expression by co-culturing HYOU1 siRNA-treated (siHYOU1) lung cancer cells with HUVECs, and treating the resulting spheroids with or without gefitinib or cisplatin. After MCTSs were allowed to form for $72 \mathrm{~h}$, we assessed cell viability by measuring the levels of the apoptosis marker cleaved caspase-3. We observed that the suppression of HYOU1 expression in lung cancer cells caused an increase in the expression of cleaved caspase-3 in MCTSs [Figure 3-D]. These results show that depletion of HYOU1 in lung cancer cells affects the drug sensitivity of the TME.

Depletion of HYOU1 inhibits tumor growth, and the stemness and expression of EMT-related proteins in lung cancer cells

Because HYOU1 is an ER-associated chaperone induced by hypoxia [34-36], we investigated its expression in lung cancer spheroids, which exhibit hypoxia. Spheroid (3D) cultures derived from various lung cancer cells exhibited increased expression of HYOU1 relative to monolayer (2D) cultures [Figure 4A].

According to analysis of data from The Cancer Genome Atlas program, HYOU1 is an unfavorable prognostic marker in renal and thyroid cancer, but is not prognostic in lung cancer. HYOU1 has a low specificity for expression in the lung cancer TME. Nevertheless, we examined whether HYOU1 controls lung cell growth to investigate the potential effects of altered HYOU1 expression on lung growth. Clonogenic survival was diminished by depletion of HYOU1 in H460 cells (40\%), A549 cells (64.2\%), and H1299 cells (46.2\%) [Figure 4-B].

To elucidate the functional roles of HYOU1, we next examined whether HYOU1 controls tumor growth and metastasis in a spheroid model similar to the lung cancer TME.

The siRNA-mediated depletion of HYOU1 significantly increased cell death in $\mathrm{H} 460$, A549, and $\mathrm{H} 1299$ spheroids [Figure 4-C]. Expression of two apoptosis markers, cleaved PARP and cleaved caspase-3, were measured in $\mathrm{H} 460$ and A549 spheroids following HYOU1 depletion. The p38 MAPK is related to hypoxiainduced apoptosis $[37,38]$ and ERK/MAPK is involved in resistance to apoptosis under hypoxic conditions [39, 40]. Inhibition of HYOU1 expression induced p38 activation, but attenuated ERK activation, in $\mathrm{H} 460$ and A549 spheroids. However, suppressing HYOU1 expression did not alter the activation of p53 or HIF1 [Figure 4-D].

To identify the possible effects of HYOU1 on cancer stem cell (CSC) populations of lung cancer cells, HYOU1-depleted lung cancer cells were cultured under spheroid-forming conditions, and the resulting 
spheroid number and size were analyzed [Figure 5-A]. Inhibition of HYOU1 significantly attenuated the spheroid-forming capacity of lung cancer cells. As CD133 expression plays a critical role in the maintenance of stem-like properties in lung cancer [41, 42], depletion of HYOU1 decreased the expression of CD133 in lung cancer cells [Figure 5-B]. These results show that HYOU1 may also be involved in the propagation of CSCs in lung cancer.

Because CSCs have been associated with tumor initiation, therapeutic resistance, and metastasis, we next sought to determine whether inhibition of HYOU1 expression sensitizes lung cancer cells to anticancer therapies and thus enhances their efficacy. After lung cancer spheroids ( $\mathrm{NCl}-\mathrm{H} 460$ or A549) with or without siHYOU1 were subjected to gefitinib treatment, the intensity of EthD-1 staining in spheroids was measured. Depletion of HYOU1 expression markedly enhanced lung cancer spheroid chemosensitivity to gefitinib [Figure 5-C]. To investigate the effects of HYOU1 on cell migration, we also performed wound healing assays following HYOU1 depletion in lung cancer cells and found that lung cancer cell migration was attenuated by HYOU1 depletion [Figure 5-D]. We next measured the expression of epithelial-to-mesenchymal transition (EMT)-related proteins in HYOU1-depleted lung cancer cells. Expression of N-cadherin, a-SMA, vimentin, and collagen I were all decreased in HYOU1-depleted lung cancer cells [Figure 5-E]. These results demonstrate that HYOU1 plays a pivotal role not only in inhibition of tumor growth and stemness but also in enhancing the anticancer efficacy of the lung cancer TME.

\section{HYOU1 controls tumor growth via the alteration of interferon signaling in lung cancer cells}

To reveal the molecular mechanism by which HYOU1 affects lung tumor growth, we performed gene expression profiling on the HYOU1-depletion system in $\mathrm{H} 460$ cells. Using a fold difference cutoff of greater than 2.5-fold, we identified 44 genes that were differentially expressed between HYOU1-depleted H460 cells and normal H460 cells [Supplementary Table II, III]. According to the Reactome Pathway Database, HYOU1 is functionally involved in the UPR, chromatin organization, and interferon (IFN) signaling [Figure 6-A]. It is noticeable that expression of IFN-type I (IFN- $\alpha, \beta)$ were more increased than IFN-type II (IFN- $\gamma$ ) by depletion of HYOU1 expression. IFN- $\alpha, \beta$ have been found to be effective in reducing the growth of various tumor [41]. Western analysis also showed that expression of IFN-type I (IFN- $\alpha, \beta)$ were increased by inhibition of HYOU1 expression during process of cell death in lung cancer cells [Figure 6-B].

HYOU1 expression is downregulated by the inhibition of the PI3K/AKT/mTOR pathway Although HYOU1 is a larger protein than GRP78, its overall structure is highly homologous to that of GRP78. Because the inhibition of the PI3K/AKT/mTOR signaling pathway suppresses GRP78 expression [42, 43], we evaluated the effect of activating the PI3K/AKT/mTOR pathway on HYOU1 expression. Treatment with the mTOR inhibitors Torin2 and WYE-132, and the potent PI3K inhibitors GDC-0032 and PKI-402, significantly inhibited HYOU1 expression in H460 and H1299 cells [Figure 7-A].

Because mTOR inhibitors could decrease an HYOU1 expression in $\mathrm{H} 460$ and $\mathrm{H} 1299$ cells, we next examined whether mTOR controls HYOU1 expression by using siRNAs for mTOR and HYOU1 in H460 and 
H1299 cells. Western blot analysis revealed that siRNA against mTOR efficiently depleted HYOU1 expression, whereas the inhibition of HYOU1 did not affect mTOR expression [Figure 7-B] and H1299 cells. These resutls suggest that mTOR could regulate the expression of HYOU1 in lung cancers.

\section{Discussion}

ECs are the most frequently studied components of the TME. Generally, ECs undergo a phenotypic transformation to activated myofibroblast-like cells through the EndMT [20]. Cancer cells stimulate the activation of the EndMT in ECs, and transformed ECs support cancer progression by secreting diverse cytokines, growth factors, and proteins of the ER membrane protein complex. Secretomics-the analysis of the secretome (all the secreted proteins of a cell, tissue, or organism)-is important to the discovery of cancer biomarkers because secreted proteins facilitate communication between distinct cells in multicellular organisms and control a broad range of physiological functions [44]. Because CM obtained from the co-culture of lung cancer cells and HUVECs promoted robust chemoresistance in lung cancer spheroids [Figure 1], we ascertained that the paracrine effects of ECs on lung cancer chemoresistance should be further investigated in 3D culture conditions to identify the chemoresistance-inducing factor(s) present in the co-cultured $\mathrm{CM}$.

Expression of many genes, especially genes related to tRNA aminoacylation and the UPR, was highly increased in lung cancer spheroids following treatment with CM from co-cultured lung cancer cells and HUVECs [Supplementary Table I]. In the present study, we focused specifically on the functional roles of HYOU1 in lung cancer cells.

HYOU1, which is a well-characterized ER chaperone and the largest glucose-regulated protein, has an ADP-ATP exchange function via its interaction with GFP78 [45], enabling it to protect cancer cells from cell death by delaying the onset of the UPR and binding to ER stress sensors. HYOU1 has also been linked to cancer development and progression through its facilitation of chemoresistance, tumor invasion, and angiogenesis in various tumor types. Conversely, HYOU1 has been shown to have potent antitumor effects in vivo dependent on cytotoxic $\mathrm{CD} 8^{+} \mathrm{T}$ cells, so it may be an effective target in a new vaccine platform to generate a therapeutic antitumor response [26]. Given these conflicting data, the functions of HYOU1 remain controversial and must be studied in the TME.

In our study, hypoxia increased the expression of HIF1 and HYOU1 in lung tumor spheroids, whereas silencing of hypoxia-induced HYOU1 suppressed tumor growth [Figure 4]. CSC populations can cause tumor recurrence, metastasis, and treatment failure in patients with lung cancer. Hypoxia stimulates the propagation of CSC populations and triggers increased expression of HYOU1[46]. In the present study, depletion of HYOU1 expression not only suppressed the propagation of CSCs via the inhibition of CD133 expression but also regulated metastasis and chemoresistance in lung cancer cells [Figure 5]. Hence, we consider HYOU1 to be an attractive target for lung cancer therapeutics. 
IFN- $\alpha, \beta$ induced apoptotic cell death in various tumors and also in triggering the anti-tumor immune response in humans $[41,47-50]$. In lung cancer cells, inhibition of HYOU1 increased the expression IFN- $a$ and IFN- $\beta$ during cell death [Figure 6]. Although GRPs are mainly induced in response to ER stress, induction of HYOU1 in spheroids co-cultured with ECs and NSCLC cells was not dependent on the ER stress response [Figure 3B]. Because GRPs are also mediated by PI3K/AKT/mTOR signaling [42, 51, 52], we tested the effects of mTOR and PI3K inhibitors on the expression of HYOU1 in lung cancer cells. Inhibition of PI3K/AKT/mTOR signaling inhibited HYOU1 expression in lung cancer cells [Figure 7]. Increases in both HYOU expression and pAKT (Ser473)/mTOR occurred simultaneously in H460 spheroids grown in CM from lung cancer cells co-cultured with ECs and in MCTSs co-cultured with ECs and NSCLC cells [Figure 2-D, 3-B]. We showed that induction of HYOU1 via the activation of $\mathrm{PISK} / \mathrm{AKT} / \mathrm{mTOR}$ signaling facilitates tumor malignancy in lung cancer. HYOU1 also promotes cell growth and metastasis by modulating the PI3K/AKT pathway in epithelial ovarian cancer [53]. Several mTOR-targeted agents are under clinical development for the treatment of lung cancer [54].

\section{Conclusions}

In this study, we sought to elucidate the mechanisms underlying HYOU1-induced chemoresistance in MCTSs co-cultured with ECs and NSCLC cells. Our study demonstrated that depletion of HYOU1 suppresses tumor growth, chemoresistance, and migration via inhibiting CSC populations by increasing the expression of IFN- $\alpha$ and IFN- $\beta$ in lung cancer cells. Expression of HYOU1 is modulated by the activation of the PI3K/AKT/mTOR pathway [Figure 8]. Hence, selective inhibitors of HYOU1 expression could represent promising therapeutic targets for overcoming chemoresistance and tumorigenesis in lung cancer.

\section{Abbreviations}

TME

Tumor microenvironments

MCTSs

Multicellular tumor spheroids

ECs

Endothelial cells

HYOU1

Hypoxia up-regulated protein 1

NSCLC

Non-small-cell lung cancer cells

HUVEC

Human Umbilical Vein Endothelial Cells

2D

Two dimensional 
3D

Three dimensional

$\mathrm{CM}$

Conditioned medium

$\mathrm{PI} 3 \mathrm{~K}$

Phosphatidylinositol-4,5-bisphosphate 3-kinase

a-SMA

a-smooth muscle actin

EthD-1

Ethdium homodimer-1

IFN

Interferon

mTOR

Mammalian target of rapamycin

\section{Declarations}

\section{Consent for publication}

All authors read and approved the final manuscript for publication.

\section{Availability of data and materials}

Information is included in the Methods section.

\section{Competing interests}

The authors declare that they have no competing interests.

\section{Funding}

This work was supported by the National Research foundation of Korea (NRF) grant funded by the Korea government (MSIP) (2017M3A9G7072864 and NRF-2017M3A9G6068246) and Gyeonggi-do

Authors' contributions

$\mathrm{ML}$ and YS designed the in vitro experiments, analyzed data and prepared the manuscript. IC performed microarray and bioinformatics analysis. SK and SL performed forming of multicellular tumor spheroids for this study. SK and JK were involved in drug screening to identify HYOU1 inhibitors. HS designed and was the overseer of the entire study. 


\section{Acknowledgments}

The authors would like to thank Joo Hwan No, team head of leishmania Research laboratory, Institut Pasteur Korea, for providing of mTOR inhibitors.

\section{References}

1. Lynch TJ, Bell DW, Sordella R, Gurubhagavatula S, Okimoto RA, Brannigan BW, Harris PL, Haserlat SM, Supko JG, Haluska FG, et al. Activating mutations in the epidermal growth factor receptor underlying responsiveness of non-small-cell lung cancer to gefitinib. $\mathrm{N}$ Engl $\mathrm{J}$ Med. 2004;350(21):2129-39.

2. Zappa C, Mousa SA. Non-small cell lung cancer: current treatment and future advances. Transl Lung Cancer Res. 2016;5(3):288-300.

3. Pao W, Miller V, Zakowski M, Doherty J, Politi K, Sarkaria I, Singh B, Heelan R, Rusch V, Fulton L, et al. EGF receptor gene mutations are common in lung cancers from "never smokers" and are associated with sensitivity of tumors to gefitinib and erlotinib. Proc Natl Acad Sci U S A. 2004;101(36):1330611.

4. Camidge DR, Pao W, Sequist LV. Acquired resistance to TKIs in solid tumours: learning from lung cancer. Nature reviews Clinical oncology. 2014;11(8):473-81.

5. Yu HA, Arcila ME, Rekhtman N, Sima CS, Zakowski MF, Pao W, Kris MG, Miller VA, Ladanyi M, Riely GJ. Analysis of tumor specimens at the time of acquired resistance to EGFR-TKI therapy in 155 patients with EGFR-mutant lung cancers. Clinical cancer research: an official journal of the American Association for Cancer Research. 2013;19(8):2240-7.

6. Shaw AT, Solomon B. Targeting anaplastic lymphoma kinase in lung cancer. Clin Cancer Res. 2011;17(8):2081-6.

7. Della Corte CM, Viscardi G, Di Liello R, Fasano M, Martinelli E, Troiani T, Ciardiello F, Morgillo F. Role and targeting of anaplastic lymphoma kinase in cancer. Mol Cancer. 2018;17(1):30.

8. Joshi A, Pande N, Noronha V, Patil V, Kumar R, Chougule A, Trivedi V, Janu A, Mahajan A, Prabhash K. ROS1 mutation non-small cell lung cancer-access to optimal treatment and outcomes. Ecancermedicalscience. 2019;13:900.

9. Shaw AT, Solomon BJ, Chiari R, Riely GJ, Besse B, Soo RA, Kao S, Lin CC, Bauer TM, Clancy JS, et al. Lorlatinib in advanced ROS1-positive non-small-cell lung cancer: a multicentre, open-label, singlearm, phase 1-2 trial. Lancet Oncol. 2019;20(12):1691-701.

10. Farago AF, Taylor MS, Doebele RC, Zhu VW, Kummar S, Spira Al, Boyle TA, Haura EB, Arcila ME, Benayed R, et al: Clinicopathologic Features of Non-Small-Cell Lung Cancer Harboring an NTRK Gene Fusion. JCO Precis Oncol 2018, 2018.

11. Roskoski R Jr. Properties of FDA-approved small molecule protein kinase inhibitors: a 2020 update. Pharmacol Res 2019:104609. 
12. Laetsch TW, DuBois SG, Mascarenhas L, Turpin B, Federman N, Albert CM, Nagasubramanian R, Davis JL, Rudzinski E, Feraco AM, et al. Larotrectinib for paediatric solid tumours harbouring NTRK gene fusions: phase 1 results from a multicentre, open-label, phase 1/2 study. Lancet Oncol. 2018;19(5):705-14.

13. Marchetti A, Felicioni L, Malatesta S, Grazia Sciarrotta M, Guetti L, Chella A, Viola P, Pullara C, Mucilli F, Buttitta F. Clinical features and outcome of patients with non-small-cell lung cancer harboring BRAF mutations. J Clin Oncol. 2011;29(26):3574-9.

14. Baik CS, Myall NJ, Wakelee HA. Targeting BRAF-Mutant Non-Small Cell Lung Cancer: From Molecular Profiling to Rationally Designed Therapy. Oncologist. 2017;22(7):786-96.

15. Zito Marino F, Bianco R, Accardo M, Ronchi A, Cozzolino I, Morgillo F, Rossi G, Franco R. Molecular heterogeneity in lung cancer: from mechanisms of origin to clinical implications. Int J Med Sci. 2019;16(7):981-9.

16. Lim SB, Yeo T, Lee WD, Bhagat AAS, Tan SJ, Tan DSW, Lim WT, Lim CT. Addressing cellular heterogeneity in tumor and circulation for refined prognostication. Proc Natl Acad Sci U S A. 2019;116(36):17957-62.

17. Joyce JA. Therapeutic targeting of the tumor microenvironment. Cancer Cell. 2005;7(6):513-20.

18. Wu SD, Ma YS, Fang Y, Liu LL, Fu D, Shen XZ. Role of the microenvironment in hepatocellular carcinoma development and progression. Cancer Treat Rev. 2012;38(3):218-25.

19. Dudley AC. Tumor endothelial cells. Cold Spring Harb Perspect Med. 2012;2(3):a006536.

20. Kim SH, Song Y, Seo HR. GSK-3beta regulates the endothelial-to-mesenchymal transition via reciprocal crosstalk between NSCLC cells and HUVECs in multicellular tumor spheroid models. J Exp Clin Cancer Res. 2019;38(1):46.

21. Song Y, Lee SY, Kim AR, Kim S, Heo J, Shum D, Kim SH, Choi I, Lee YJ, Seo HR. Identification of radiation-induced EndMT inhibitors through cell-based phenomic screening. FEBS Open Bio. 2019;9(1):82-91.

22. Choi SH, Kim AR, Nam JK, Kim JM, Kim JY, Seo HR, Lee HJ, Cho J, Lee YJ. Tumour-vasculature development via endothelial-to-mesenchymal transition after radiotherapy controls CD44v6(+) cancer cell and macrophage polarization. Nat Commun. 2018;9(1):5108.

23. Hirschhaeuser F, Menne H, Dittfeld C, West J, Mueller-Klieser W, Kunz-Schughart LA. Multicellular tumor spheroids: an underestimated tool is catching up again. J Biotechnol. 2010;148(1):3-15.

24. Song Y, Kim SH, Kim KM, Choi EK, Kim J, Seo HR. Activated hepatic stellate cells play pivotal roles in hepatocellular carcinoma cell chemoresistance and migration in multicellular tumor spheroids. Sci Rep. 2016;6:36750.

25. Yu X, Guo C, Yi H, Qian J, Fisher PB, Subjeck JR, Wang XY. A multifunctional chimeric chaperone serves as a novel immune modulator inducing therapeutic antitumor immunity. Cancer Res. 2013;73(7):2093-103.

26. Wang H, Pezeshki AM, Yu X, Guo C, Subjeck JR, Wang XY. The Endoplasmic Reticulum Chaperone GRP170: From Immunobiology to Cancer Therapeutics. Front Oncol. 2014;4:377. 
27. Park JE, Facciponte J, Chen X, MacDonald I, Repasky EA, Manjili MH, Wang XY, Subjeck JR. Chaperoning function of stress protein grp170, a member of the hsp70 superfamily, is responsible for its immunoadjuvant activity. Cancer Res. 2006;66(2):1161-8.

28. Wang XY, Kazim L, Repasky EA, Subjeck JR. Immunization with tumor-derived ER chaperone grp170 elicits tumor-specific CD8 + T-cell responses and reduces pulmonary metastatic disease. Int J Cancer. 2003;105(2):226-31.

29. Stojadinovic A, Hooke JA, Shriver CD, Nissan A, Kovatich AJ, Kao TC, Ponniah S, Peoples GE, Moroni M. HYOU1/Orp150 expression in breast cancer. Med Sci Monit. 2007;13(11):BR231-239.

30. Zhou Y, Liao Q, Li X, Wang H, Wei F, Chen J, Yang J, Zeng Z, Guo X, Chen P, et al. HYOU1, Regulated by LPLUNC1, Is Up-Regulated in Nasopharyngeal Carcinoma and Associated with Poor Prognosis. J Cancer. 2016;7(4):367-76.

31. Gao YY, Liu BQ, Du ZX, Zhang HY, Niu XF, Wang HQ. Implication of oxygen-regulated protein 150 (ORP150) in apoptosis induced by proteasome inhibitors in human thyroid cancer cells. J Clin Endocrinol Metab. 2010;95(11):E319-26.

32. Namba T, Hoshino T, Tanaka K, Tsutsumi S, Ishihara T, Mima S, Suzuki K, Ogawa S, Mizushima T. Upregulation of $150-k D a$ oxygen-regulated protein by celecoxib in human gastric carcinoma cells. Mol Pharmacol. 2007;71(3):860-70.

33. Fu Y, Lee AS. Glucose regulated proteins in cancer progression, drug resistance and immunotherapy. Cancer Biol Ther. 2006;5(7):741-4.

34. Tamatani M, Matsuyama T, Yamaguchi A, Mitsuda N, Tsukamoto Y, Taniguchi M, Che YH, Ozawa K, Hori O, Nishimura $\mathrm{H}$, et al. ORP150 protects against hypoxia/ischemia-induced neuronal death. Nat Med. $2001 ; 7(3): 317-23$.

35. Tsukamoto Y, Kuwabara K, Hirota S, Ikeda J, Stern D, Yanagi H, Matsumoto M, Ogawa S, Kitamura Y. $150-k D$ oxygen-regulated protein is expressed in human atherosclerotic plaques and allows mononuclear phagocytes to withstand cellular stress on exposure to hypoxia and modified low density lipoprotein. J Clin Invest. 1996;98(8):1930-41.

36. Ikeda J, Kaneda S, Kuwabara K, Ogawa S, Kobayashi T, Matsumoto M, Yura T, Yanagi H. Cloning and expression of cDNA encoding the human 150 kDa oxygen-regulated protein, ORP150. Biochem Biophys Res Commun. 1997;230(1):94-9.

37. Chae HJ, Kim SC, Han KS, Chae SW, An NH, Kim HM, Kim HH, Lee ZH, Kim HR. Hypoxia induces apoptosis by caspase activation accompanying cytochrome $\mathrm{C}$ release from mitochondria in MC3T3E1 osteoblasts. p38 MAPK is related in hypoxia-induced apoptosis. Immunopharmacol Immunotoxicol. 2001;23(2):133-52.

38. Park EC, Rongo C. The p38 MAP kinase pathway modulates the hypoxia response and glutamate receptor trafficking in aging neurons. Elife 2016, 5.

39. Liu L, Zhang H, Sun L, Gao Y, Jin H, Liang S, Wang Y, Dong M, Shi Y, Li Z, et al. ERK/MAPK activation involves hypoxia-induced MGr1-Ag/37LRP expression and contributes to apoptosis resistance in gastric cancer. Int J Cancer. 2010;127(4):820-9. 
40. Hartel FV, Holl M, Arshad M, Aslam M, Gunduz D, Weyand M, Micoogullari M, Abdallah Y, Piper HM, Noll T. Transient hypoxia induces ERK-dependent anti-apoptotic cell survival in endothelial cells. Am J Physiol Cell Physiol. 2010;298(6):C1501-9.

41. Ghosh D, Parida P. Interferon Therapy in Lung Cancer: Current Perspectives. Current Cancer Therapy Reviews 2017, 13.

42. Thon M, Hosoi T, Yoshii M, Ozawa K. Leptin induced GRP78 expression through the PI3K-mTOR pathway in neuronal cells. Sci Rep. 2014;4:7096.

43. Pfaffenbach KT, Pong M, Morgan TE, Wang H, Ott K, Zhou B, Longo VD, Lee AS. GRP78/BiP is a novel downstream target of IGF-1 receptor mediated signaling. J Cell Physiol. 2012;227(12):380311.

44. Meissner F, Scheltema RA, Mollenkopf HJ, Mann M. Direct proteomic quantification of the secretome of activated immune cells. Science. 2013;340(6131):475-8.

45. Andreasson C, Rampelt H, Fiaux J, Druffel-Augustin S, Bukau B. The endoplasmic reticulum Grp170 acts as a nucleotide exchange factor of Hsp70 via a mechanism similar to that of the cytosolic Hsp110. J Biol Chem. 2010;285(16):12445-53.

46. De Francesco EM, Maggiolini M, Tanowitz HB, Sotgia F, Lisanti MP. Targeting hypoxic cancer stem cells (CSCs) with Doxycycline: Implications for optimizing anti-angiogenic therapy. Oncotarget. 2017;8(34):56126-42.

47. Hobeika AC, Subramaniam PS, Johnson HM. IFNalpha induces the expression of the cyclindependent kinase inhibitor p21 in human prostate cancer cells. Oncogene. 1997;14(10):1165-70.

48. Yasuoka Y, Naomoto Y, Yamatsuji T, Takaoka M, Kimura M, Uetsuka H, Matsubara N, Fujiwara T, Gunduz M, Tanaka N, et al. Combination of tumor necrosis factor alpha and interferon alpha induces apoptotic cell death through a c-myc-dependent pathway in p53 mutant H226br non-small-cell lung cancer cell line. Exp Cell Res. 2001;271(2):214-22.

49. Makowska A, Wahab L, Braunschweig T, Kapetanakis NI, Vokuhl C, Denecke B, Shen L, Busson P, Kontny U. Interferon beta induces apoptosis in nasopharyngeal carcinoma cells via the TRAILsignaling pathway. Oncotarget. 2018;9(18):14228-50.

50. Nair S, Mayotte J, Lokshin A, Levitt M. Induction of squamous differentiation by interferon beta in a human non-small-cell lung cancer cell line. J Natl Cancer Inst. 1994;86(5):378-83.

51. Dai RY, Chen SK, Yan DM, Chen R, Lui YP, Duan CY, Li J, He T, Li H. PI3K/Akt promotes GRP78 accumulation and inhibits endoplasmic reticulum stress-induced apoptosis in HEK293 cells. Folia Biol (Praha). 2010;56(2):37-46.

52. Lee AS. Glucose-regulated proteins in cancer: molecular mechanisms and therapeutic potential. Nat Rev Cancer. 2014;14(4):263-76.

53. Li X, Zhang NX, Ye HY, Song PP, Chang W, Chen L, Wang Z, Zhang L, Wang NN. HYOU1 promotes cell growth and metastasis via activating PI3K/AKT signaling in epithelial ovarian cancer and predicts poor prognosis. Eur Rev Med Pharmacol Sci. 2019;23(10):4126-35. 
54. Ekman S, Wynes MW, Hirsch FR. The mTOR pathway in lung cancer and implications for therapy and biomarker analysis. J Thorac Oncol. 2012;7(6):947-53.

\section{Figures}

\section{Figure 1.}

A.

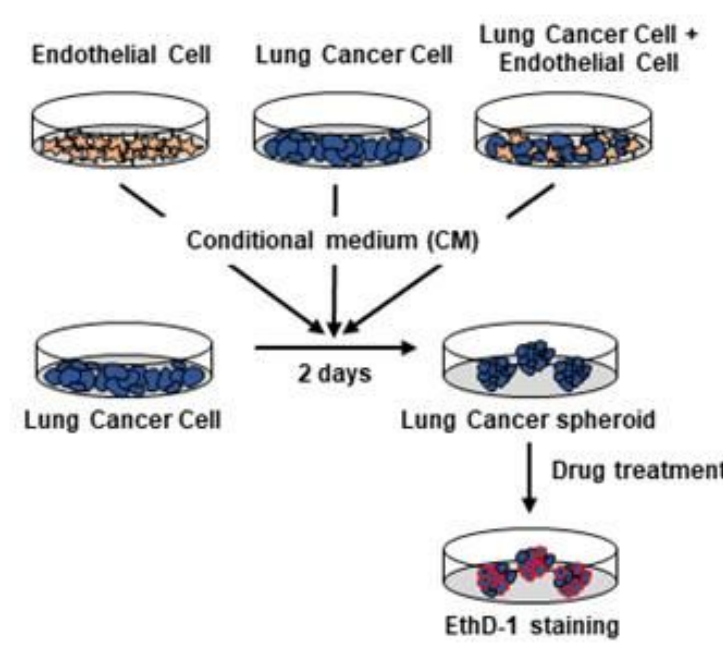

C.

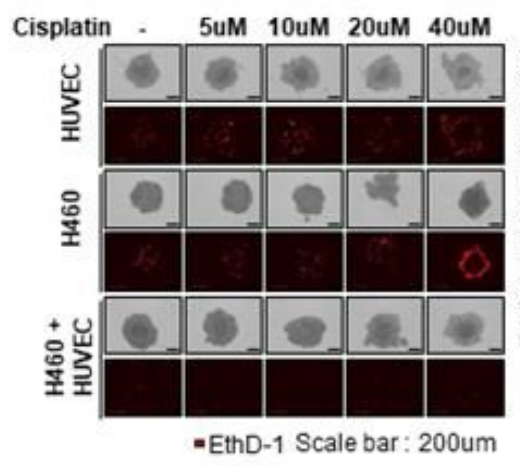

B.
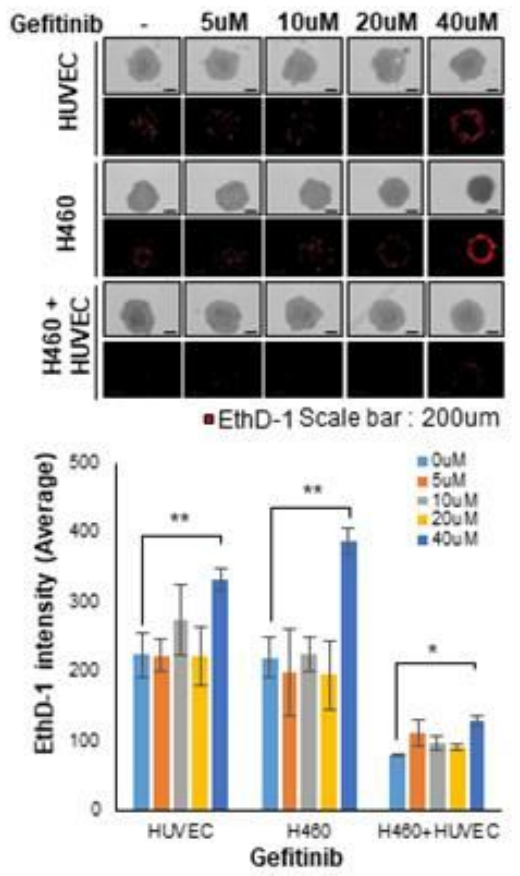
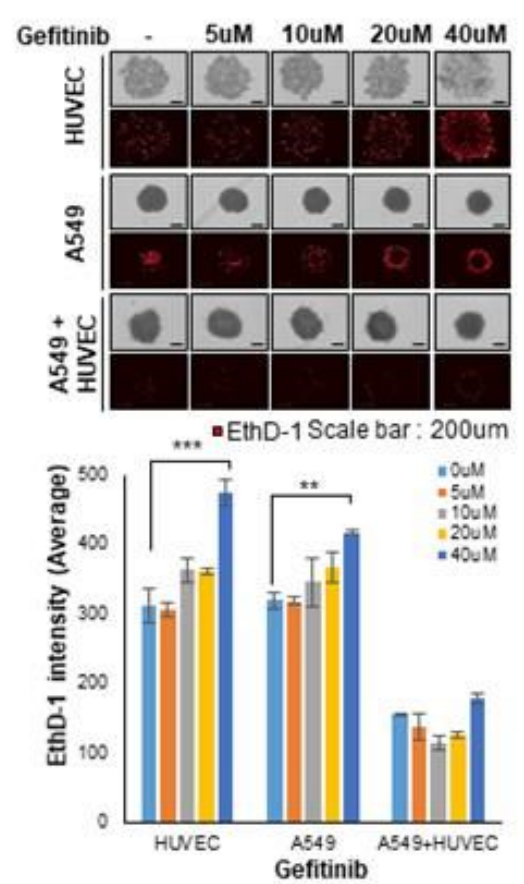
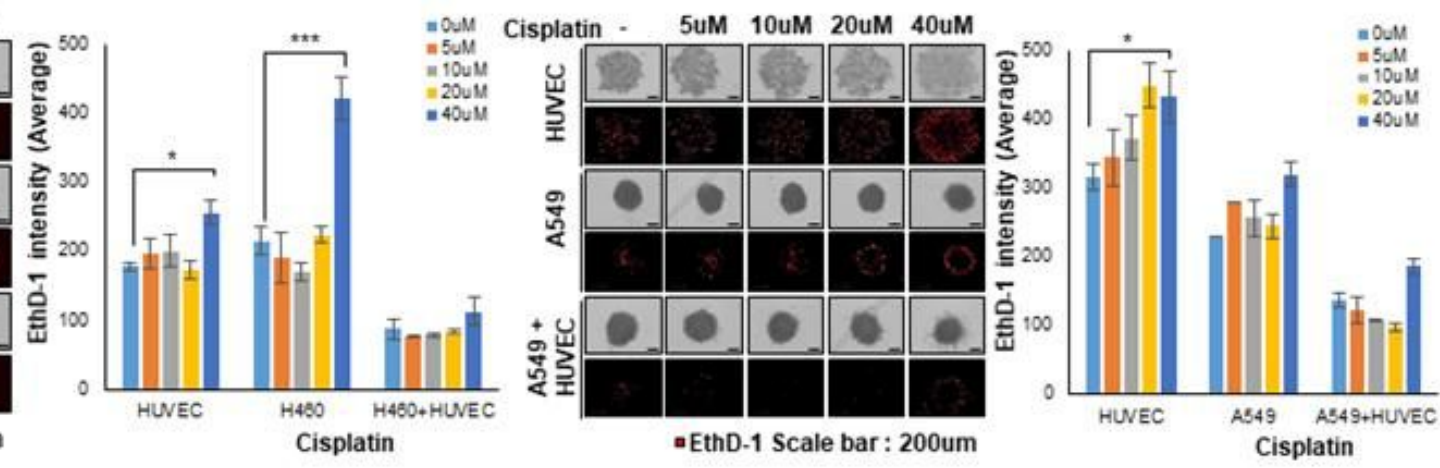

\section{Figure 1}

Crosstalk between ECs and lung cancer cells causes chemoresistance in lung cancer spheroids. (A) Experimental schematic of CM obtained from cultured HUVECs (ECs), lung cancer cells (NCI-H460 or A549), and lung cancer cells co-cultured with HUVECs. The cells were cultured for 3 days in 2D culture conditions using the same number of cells and the same amount of media. Next, three different CM were added to lung cancer cells in 3D culture conditions for 2 days. The spheroids were then treated with drugs for 2 days, and EthD-1 staining was used to identify dead cells. (B and C) Immunofluorescence images of lung cancer spheroids grown in CM obtained from cultured HUVECs, cultured lung cancer cells, or lung cancer cells co-cultured with HUVECs. The cells were treated with 5, 10, 20, or $40 \mu \mathrm{M}$ gefitinib (B) or cisplatin (C) for 2 days, and then stained with $4 \mu \mathrm{M}$ EthD-1. The images were obtained using the 
Operetta ${ }^{\circledR}$ High Content Screening System, and the intensity of EthD-1 staining in lung cancer spheroids relative to controls was analyzed using Harmony software. The data shown are the means \pm SD from three independent experiments; ${ }^{*} \mathrm{P}<0.05,{ }^{*} \mathrm{P}<0.01$, and ${ }^{* * * \mathrm{P}}<0.001$ compared to the control group.

\section{Figure 2.}
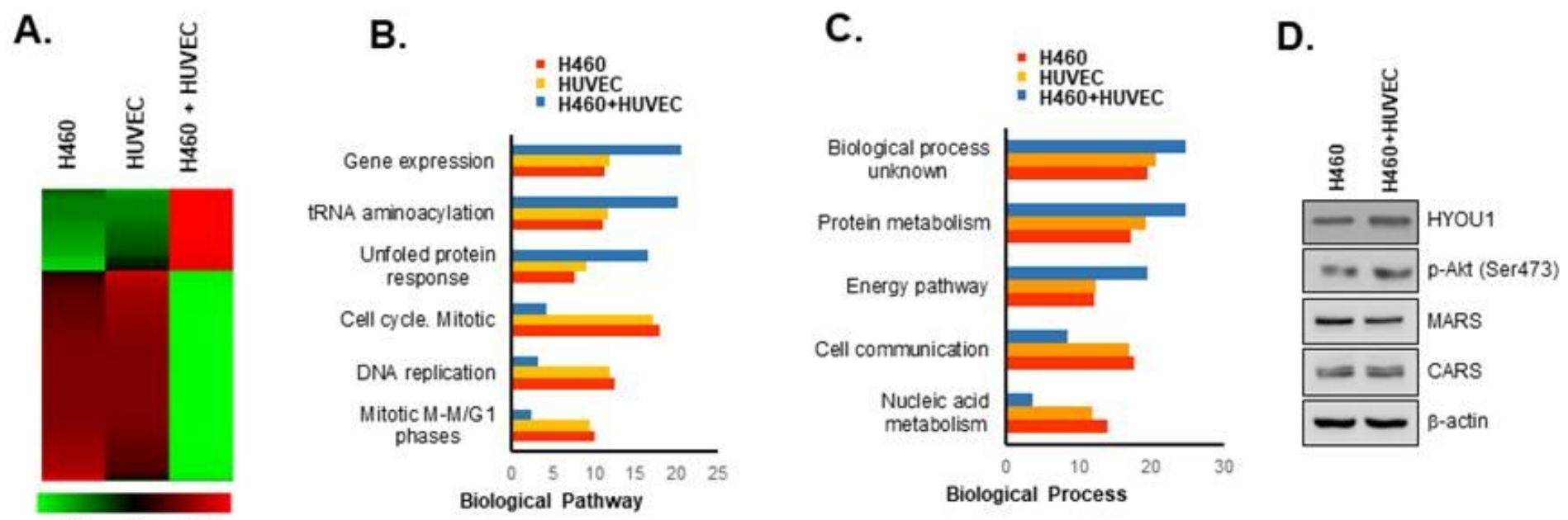

$\begin{array}{lll}1.15 & 0.0 & -1.15\end{array}$

\section{Figure 2}

HYOU1 expression is increased by factors secreted in response to crosstalk between ECs and lung cancer cells. (A) A gene expression heat map representing fold changes greater than 1.5 in samples from lung cancer spheroids grown in CM from cultured HUVECs, cultured NCl-H460 cells, or lung cancer cells cocultured with HUVECs. (B and C) Categorization of biological pathways (B) and biological processes (C) identified by microarray analysis as markedly altered in lung cancer spheroids grown in CM from cultured HUVECs, cultured NCl-H460 cells, or lung cancer cells co-cultured with HUVECs. (D) Expression levels of CARS, HYOU1, MARS, and pAKT (Ser437) in lung cancer spheroids grown in CM from NCl-H460 cells cultured alone or co-cultured with HUVECs, as assessed by western blot analysis. 


\section{Figure 3.}

A.
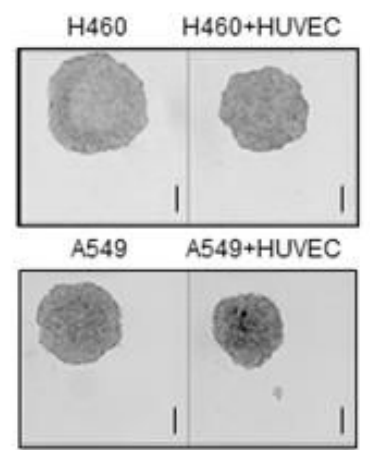

B.

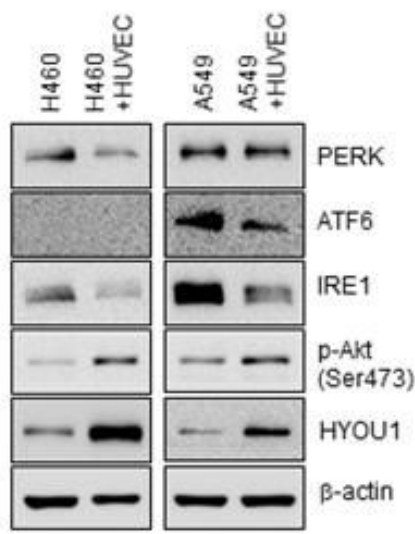

C.

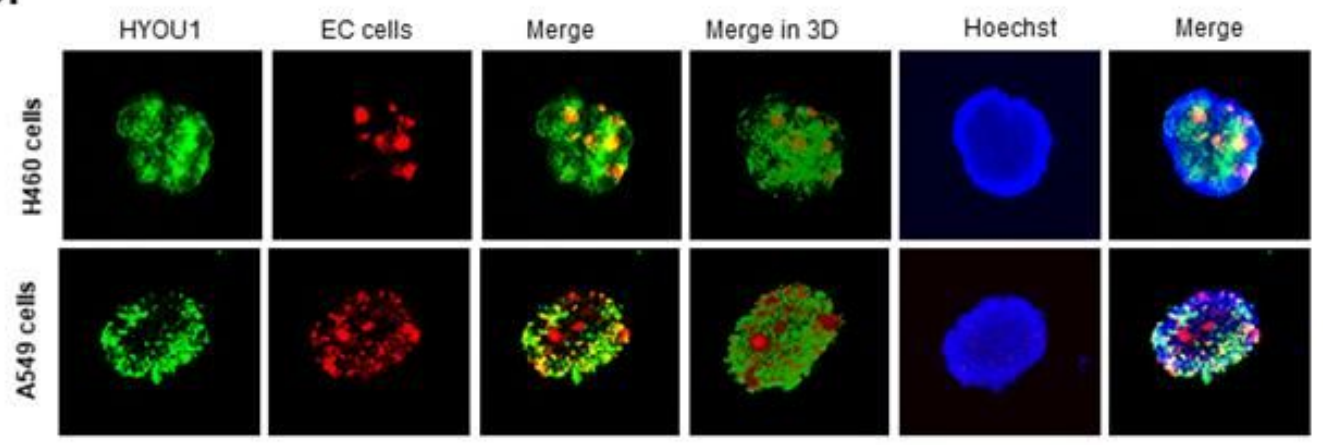

D.
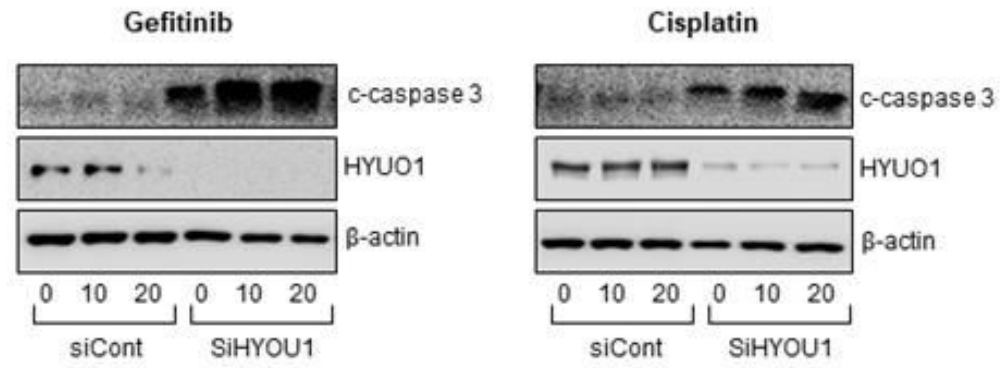

\section{Figure 3}

Reciprocal crosstalk between NSCLC cells and HUVECs causes increased HYOU1 expression in MCTSs. (A) Bright-field images of 3D spheroids co-cultured with HUVECs and NSCLC cells (NCl-H460 or A549) and spheroids cultured with lung cancer cells alone. The images were obtained using the Operetta ${ }^{\circledR}$ High Content Screening System with a 10x objective. (B) Expression levels of ATF6, HYOU1, IRE1, and pAKT (Ser473) in spheroids co-cultured with HUVECs and NSCLC cells (NCl-H460 or A549) and spheroids cultured with lung cancer cells alone, as assessed by western blot analysis. (C) Multilayer image showing immunofluorescence staining of HYOU1 in NSCLC (NCl-H460 or A549) spheroids co-cultured with HUVECs and Hoechst staining of both cell types. (D) Expression levels of cleaved caspase-3 and HYOU1 in lung cancer cells transfected with nonspecific siRNA (SiCont) or HYOU1 siRNA (SiHYOU1) and cocultured with HUVECs with or without 10 or $20 \mu \mathrm{M}$ gefitinib or cisplatin for $72 \mathrm{~h}$, as assessed by western blot analysis. 


\section{Figure 4.}

A.

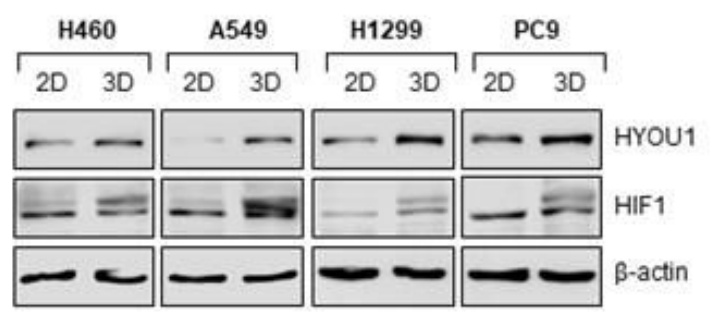

C.
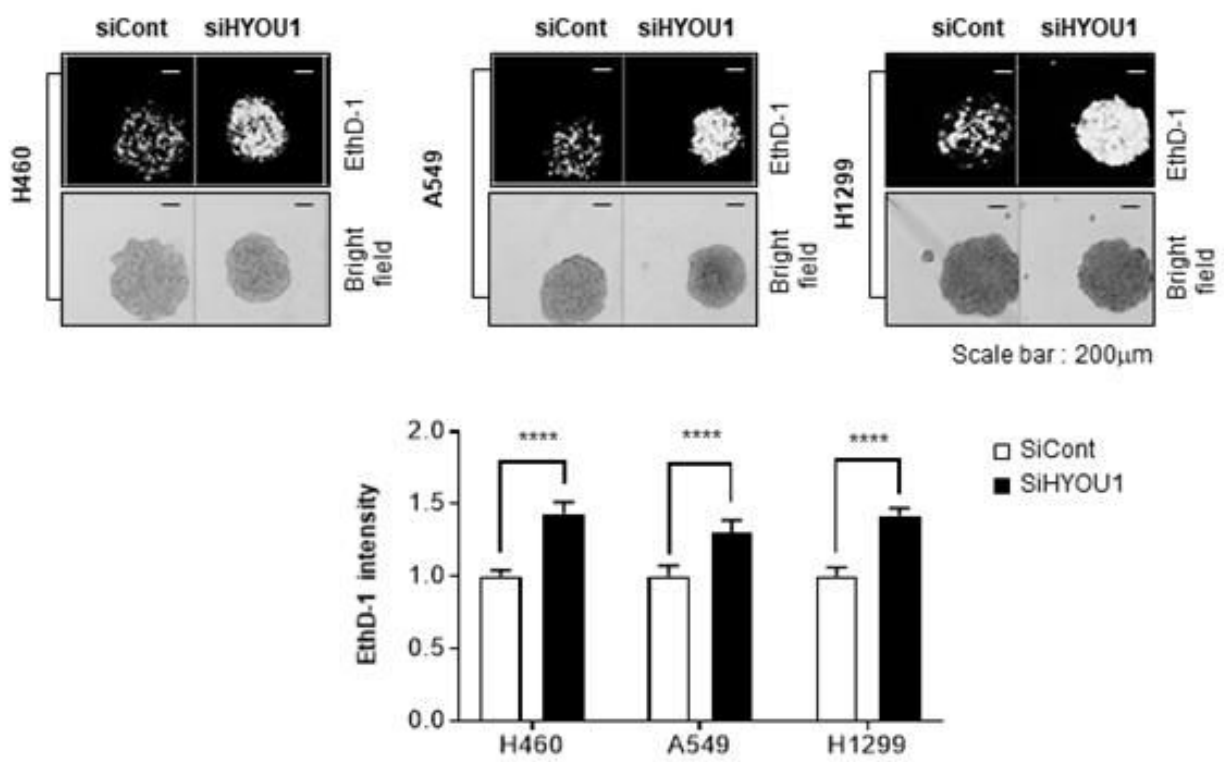

B.
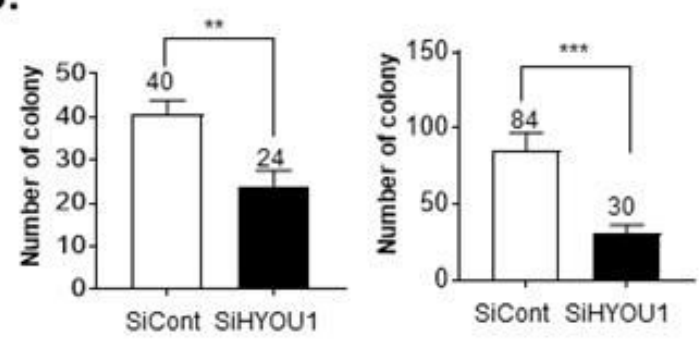

D.
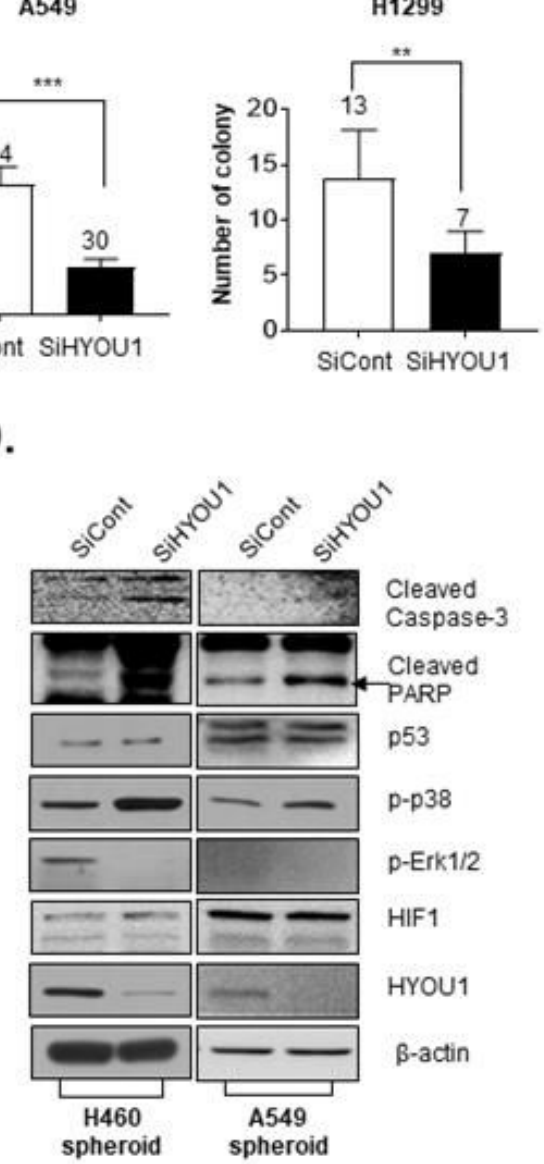

Figure 4

Depletion of HYOU1 inhibits tumor growth in lung cancer cells. (A) Expression levels of HIF1 and HYOU1 in monolayer (2D)- or spheroid (3D)-cultured NSCLC cells (NCl-H460, A549, H1299, and PC9), as assessed by western blot analysis. (B) Clonogenic survival in NSCLC cells (NCl-H460, A549, and H1299) transfected with nonspecific siRNA (SiCont) or HYOU1 siRNA (SiHYOU1), as assessed by colony formation assay. (C) Immunofluorescence and bright-field images of lung cancer spheroids ( $\mathrm{NCl}-\mathrm{H} 460, \mathrm{~A} 549$, and $\mathrm{H} 1299$ ) transfected with nonspecific siRNA (SiCont) or HYOU1 siRNA (SiHYOU1). The spheroids were stained with $4 \mu \mathrm{M}$ EthD-1. The images were obtained using the Operetta ${ }^{\circledR}$ High Content Screening System, and the intensity of EthD-1 staining in lung cancer spheroids relative to controls was analyzed using Harmony software. (D) Expression of cleaved caspase-3, cleaved PARP, HIF1, HYOU1, pp38, p53, and pErk1/2 in lung cancer spheroids (NCl-H460 and A549) transfected with nonspecific siRNA (SiCont) or HYOU1 siRNA (SiHYOU1). The data shown are the means \pm SD from three independent experiments; ${ }^{*} P<0.05$, $* * P<$ 0.01 , and $* \star * \mathrm{P}<0.001$ compared to the control group. 
A.

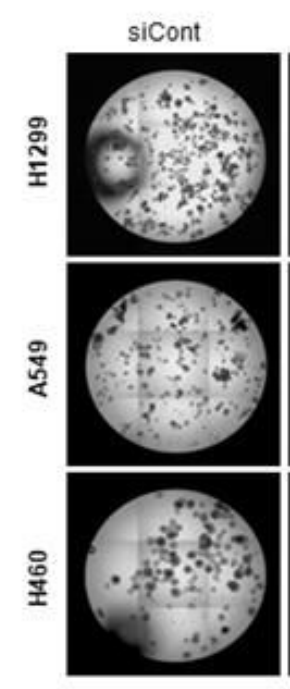

B. SiHYOU1

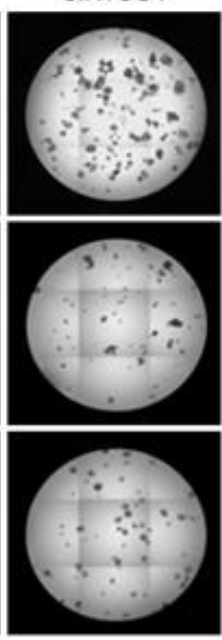

C.

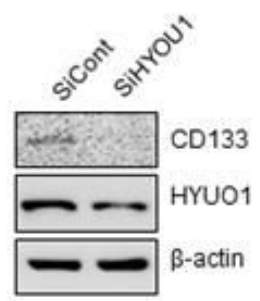

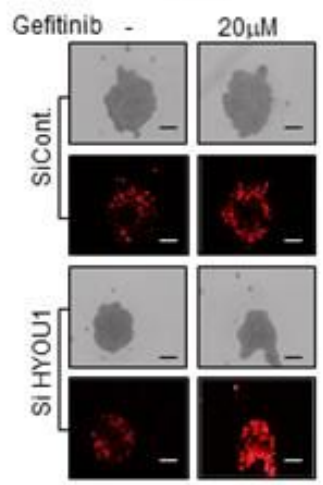

A549

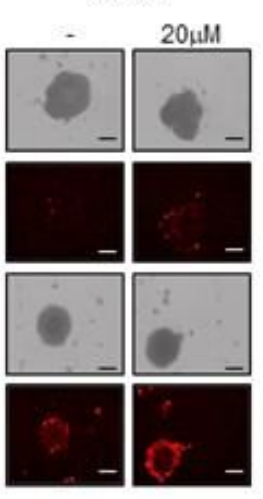

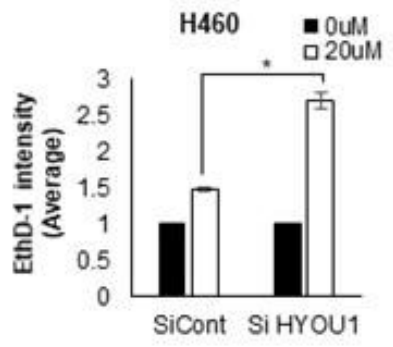

A549

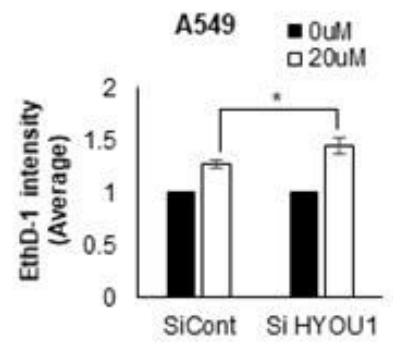

D.

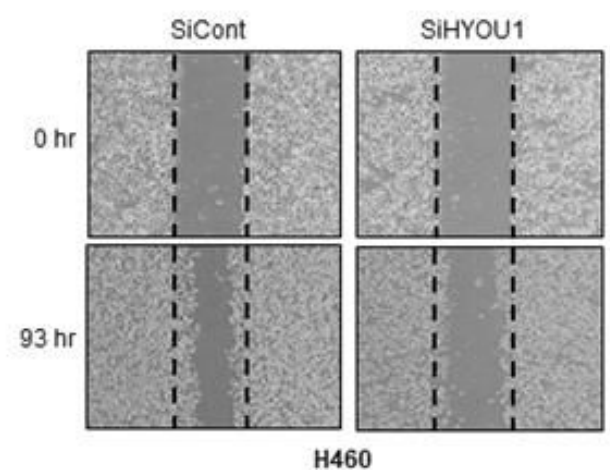

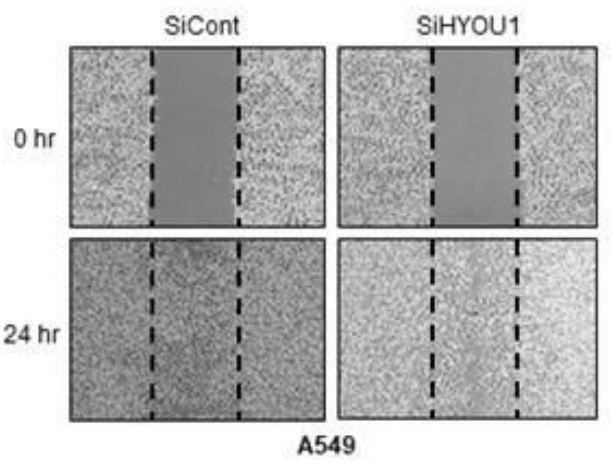

E.

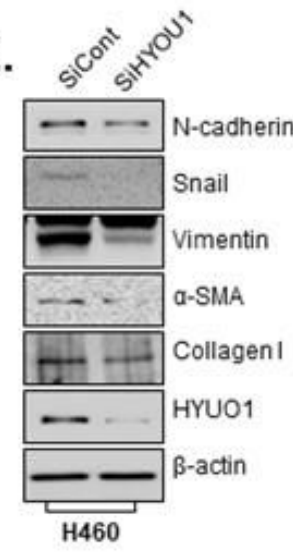

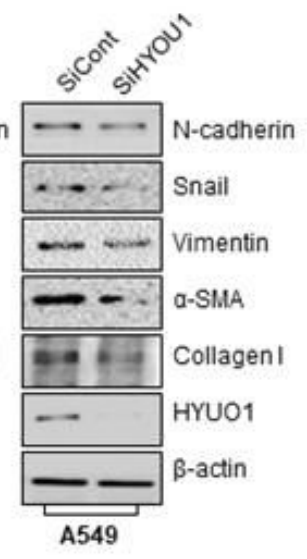

Figure 5

Depletion of HYOU1 inhibits stemness and the expression of EMT-related proteins in lung cancer cells. (A) CSC spheroid formation of $\mathrm{H} 1299$, A549 and $\mathrm{NCl}-\mathrm{H} 460$ cells transfected with nonspecific siRNA (SiCont) or HYOU1 siRNA (SiHYOU1). (B) Expression levels of CD133 and HYOU1 in H1299 cells transfected with nonspecific siRNA (SiCont) or HYOU1 siRNA (SiHYOU1). (C) Bright-field and immunofluorescence images of lung cancer spheroids (NCl-H460 and A549) transfected with nonspecific siRNA (SiCont) or HYOU1 siRNA (SiHYOU1) and treated with $20 \mu \mathrm{M}$ gefitinib. The spheroids were stained with $4 \mu \mathrm{M}$ EthD-1. The images were obtained using the Operetta ${ }^{\circledR}$ High Content Screening System, and the intensity of EthD-1 staining in lung cancer spheroids was analyzed using Harmony software. (D) Bright-field images of NSCLC cells (NCl-H460 and A549) transfected with nonspecific siRNA (SiCont) or HYOU1 siRNA (SiHYOU1), as assessed by migration assay. (E) Expression levels of ख-SMA, collagen I, HYOU1, Ncadherin, and vimentin in in lung cancer spheroids ( $\mathrm{NCl}-\mathrm{H} 460$ and A549) transfected with nonspecific siRNA (SiCont) or HYOU1 siRNA (SiHYOU1). The data shown are the means \pm SD from three independent experiments; ${ }^{*} P<0.05,{ }^{*} \mathrm{P}<0.01$, and ${ }^{* \star *} \mathrm{P}<0.001$ compared to the control group. 
Figure 6.

A.

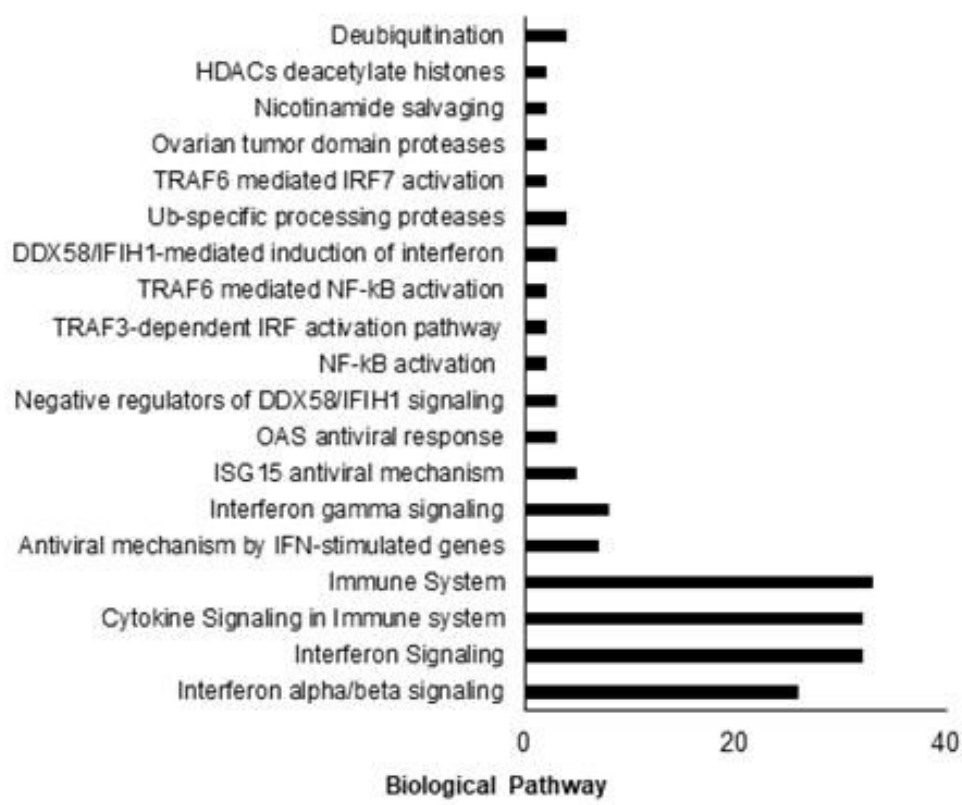

B.

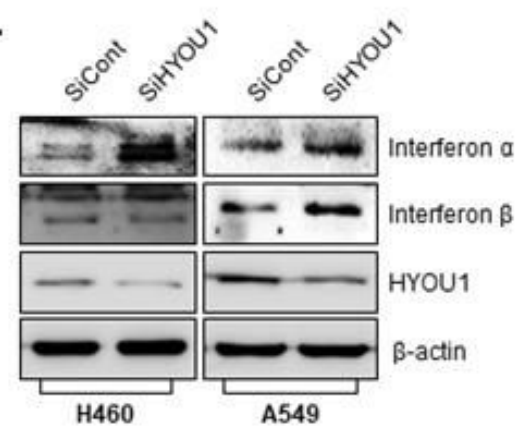

\section{Figure 6}

HYOU1 controls tumor growth via the regulation of IFN signaling in lung cancer cells. (A) Target pathways identified by microarray analysis as markedly altered in $\mathrm{NCl}-\mathrm{H} 460$ cells transfected with siHYOU1. (B) Expression levels of IFN- $a$, and IFN- $\beta$ in NSCLC cells (NCl-H460 and A549) transfected with nonspecific siRNA (SiCont) or HYOU1 siRNA (SiHYOU1). 
Figure 7.

A.

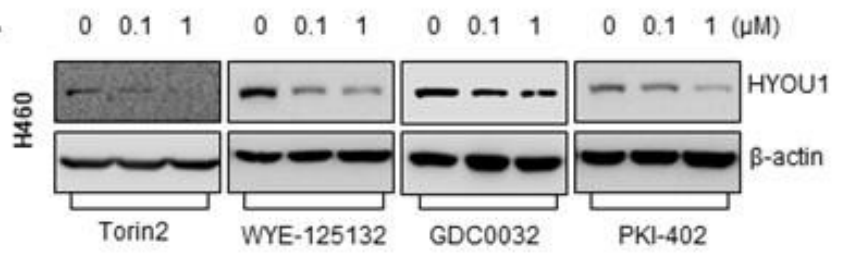

B.
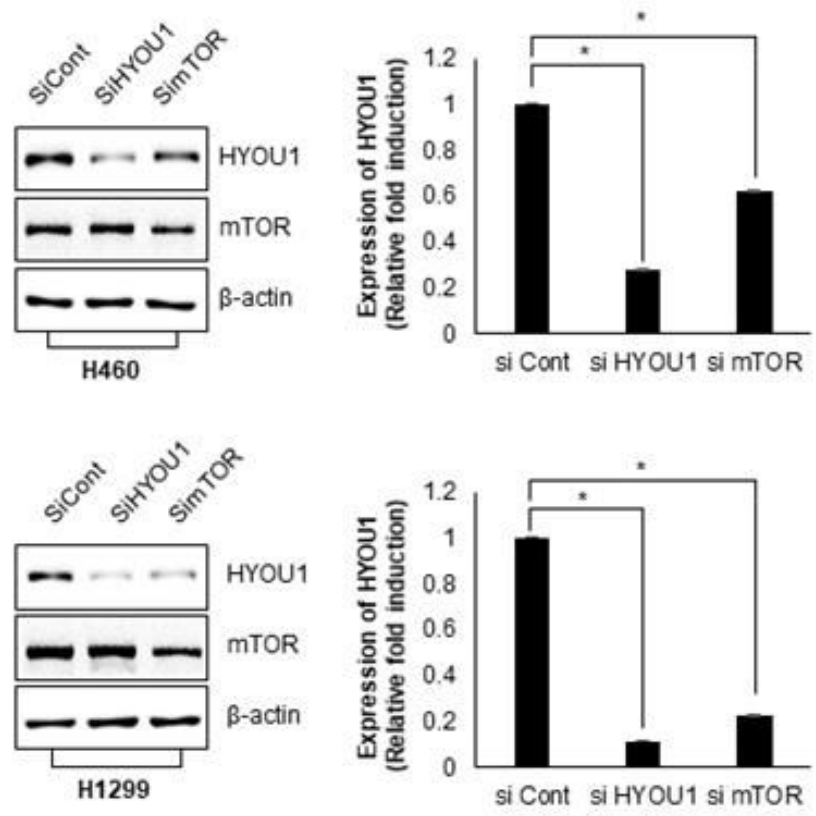
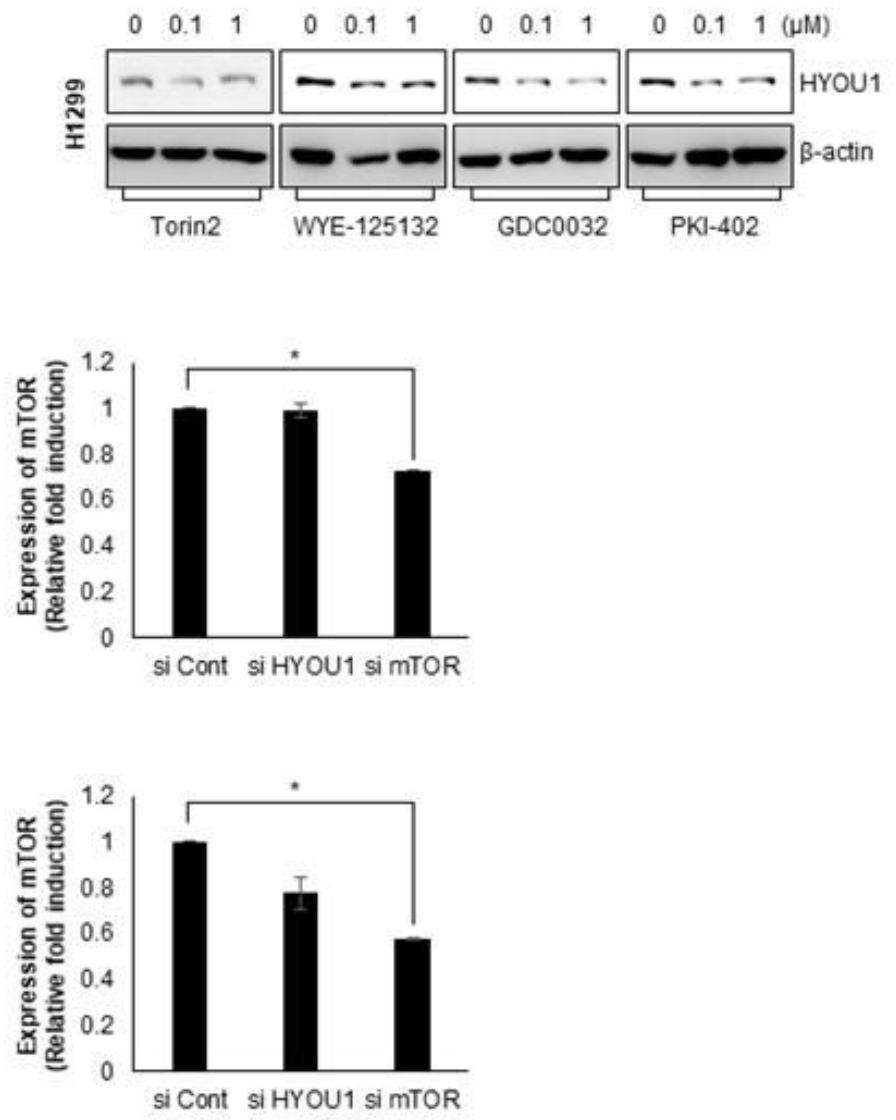

Figure 7

HYOU1 expression is downregulated by the inhibition of the PI3K/AKT/mTOR pathway. (A) Expression levels of HYOU1 in NSCLC cells (NCI-H460 and H1299) treated with 0.1 or $1 \mu \mathrm{M}$ of an mTOR inhibitor (Torin2 or WYE-125132) or a PI3K inhibitor (GDC0032 or PKI-402). (B) Expression levels of HYOU1 and mTOR in NSCLC cells (NCl-H460 and H1299) transfected with nonspecific siRNA (SiCont), HYOU1 siRNA (SiHYOU1), or mTOR siRNA (SimTOR). 


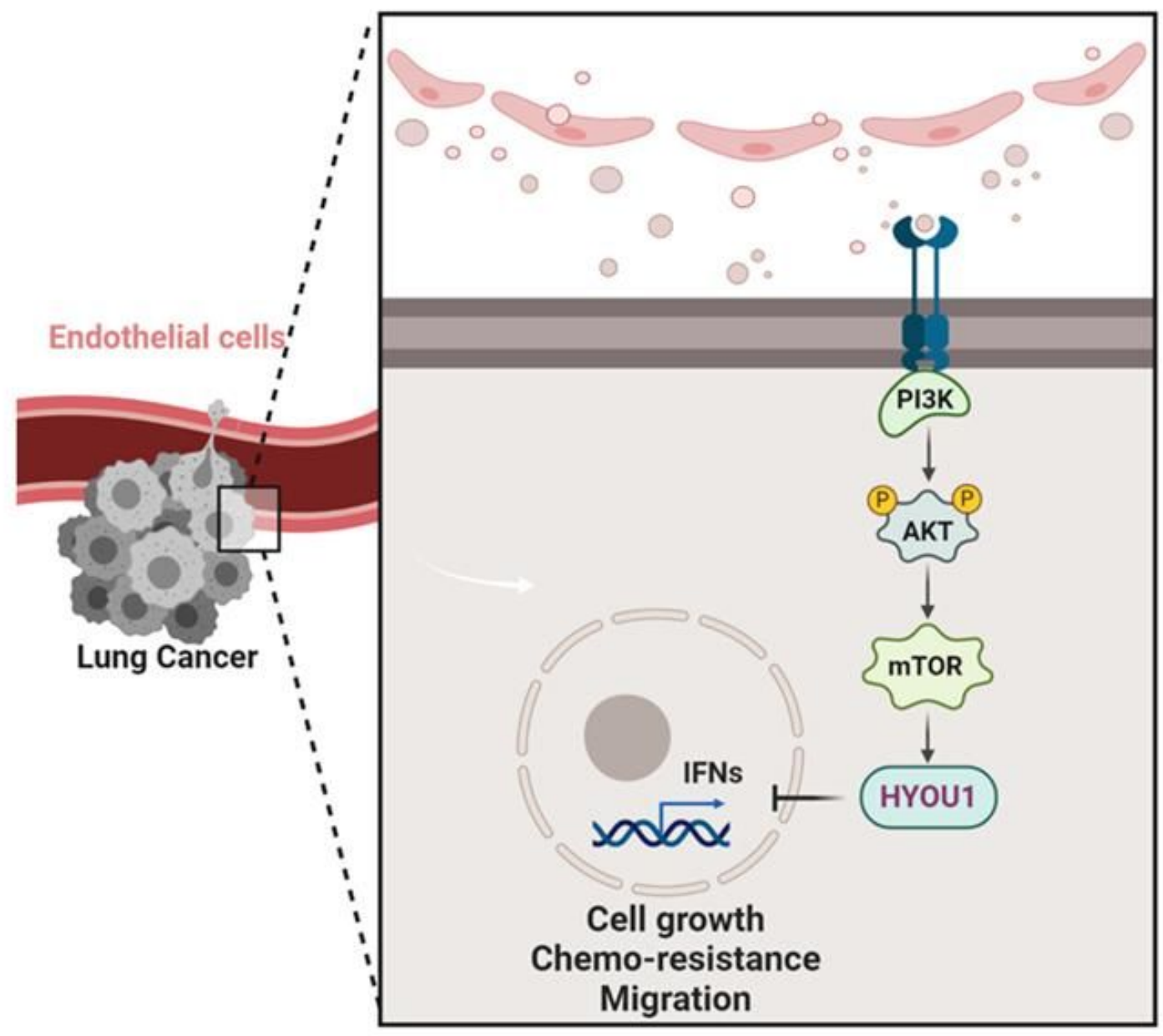

Figure 8

Schematic diagram of HYOU1 regulation in spheroids co-cultured with HUVECs and NSCLC cells. Cell growth, chemoresistance, and migration in lung cancer spheroids are up-regulated by co-culturing with HUVECs (ECs) and lung cancer (NSCLC) cells.

\section{Supplementary Files}

This is a list of supplementary files associated with this preprint. Click to download.

- supplementarydata.pdf 